\title{
Minimal Elastographic Modeling of Breast Cancer for Model Based Tumor Detection in a Digital Image based Elasto Tomography (DIET) System
}

Thomas F Lotz, Natalie Muller, Christopher E Hann and J Geoffrey Chase

Centre for Bioengineering, Department of Mechanical Engineering

University of Canterbury, Christchurch, New Zealand

Contact: thomas.lotz@canterbury.ac.nz

\section{Background}

- Breast cancer claims over 400,000 annual deaths worldwide [1]

- X-ray mammography is the only accepted screening modality

- Mammography is controversial due to low compliance, poor sensitivity [2], radiation exposure [3] and discomfort [4]

-A less invasive and low cost method is required to extend screening programs to currently underserved populations, i.e. in developing countries

-This research presents a model-based filter of surface vibrations to estimate tissue stiffness within the breast

\section{DIET - Digital Image based Elasto Tomography}

- Elastographic approach to detect areas of higher stiffness in the breast

- Non-invasive, pain free (no compressions), low cost, portable

- High stiffness contrast of $300 \%-1500 \%$ between healthy and cancer tissue [5], compared to $10-20 \%$ x-ray contrast

- Track surface motion of oscillating breast $(5-100 \mathrm{~Hz})$ to derive elastic properties [6]

- Inverse solution with Finite Element Models deliver size and location of tumour [6], at high computational cost.

- Faster model based diagnostic required for real-time diagnostic performance
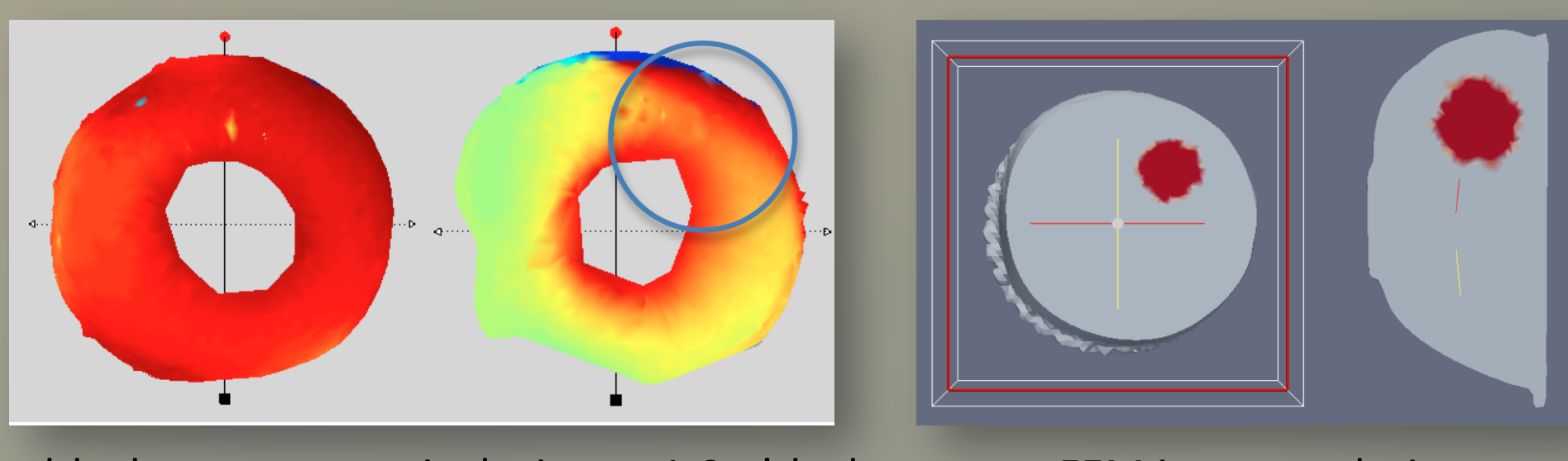

Healthy breast tumor inclusion at 1-2 o'clock

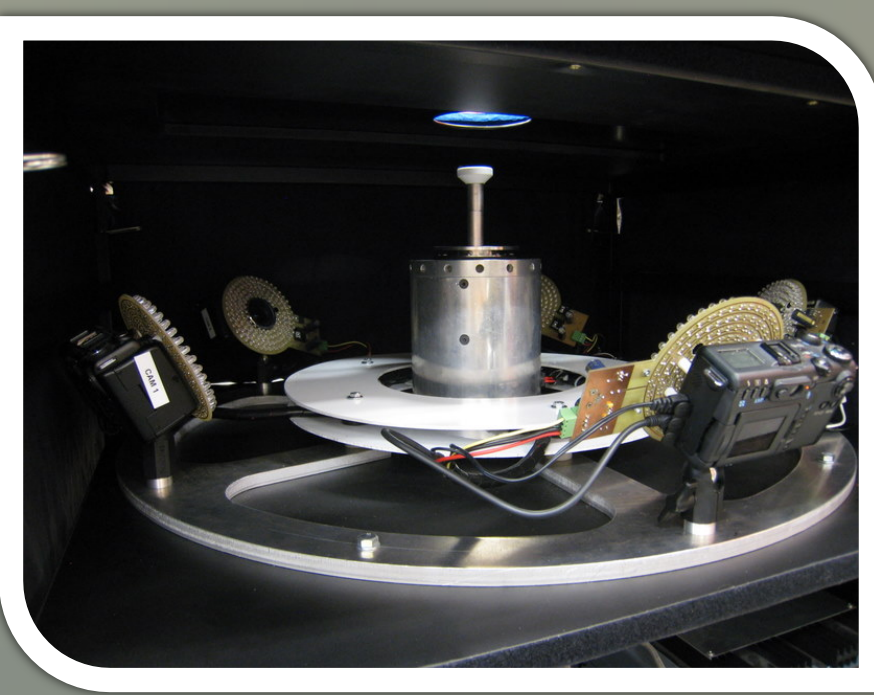

FEM inverse solution

DIET imaging system

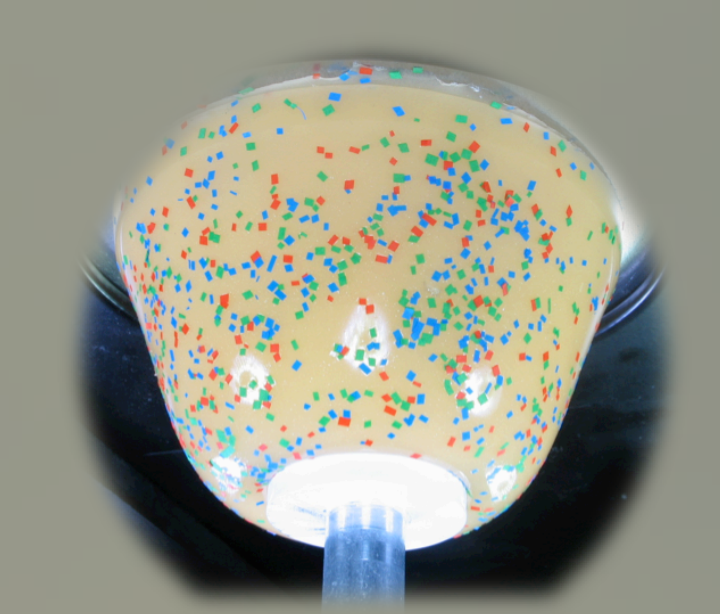

Breast phantom with markers

\section{References}

[1] S. S. Coughlin, et al. "Breast cancer as a global health concern," Cancer epidemiology, vol. 33, no. 5, pp. 315-8, 2009.

[2] M. Kriege et al., "Efficacy of MRI and mammography for breast-cancer screening in women with a familial or genetic predisposition," NEJM, 351(5), pp. 427-37, 2004. [3] R. Fazel, et al., "Exposure to low-dose ionizing radiation from medical imaging procedures," NEJM, 361(9), pp. 849-57, 2009.

[4] A. Asghari, et al., "Pain during mammography: the role of coping strategies," Pain, 108 (1-2), pp. 170-9, 2004.

[5] A. Samani, et al., "Elastic moduli of normal and pathological human breast tissues: an inversion-technique-based investigation of 169 samples," Physics in medicine and biolog 52(6), pp. 1565-76, 2007

[6] A. Peters, et al., "Estimating elasticity in heterogeneous phantoms using digital image elasto-tomography," Medical \& biological engineering \& computing, 47(1), pp.67-76, 2009.

\section{Methodology}

\section{Breast tissue Elastography by analysis of the} frequency response of its oscillating surface

1. Imaging performed with DIET system on 2 silicone breast phantoms, one healthy and one with a stiffer ( $6 x$ contrast) inclusion of $20 \mathrm{~mm}$, at a frequency range of $8-50 \mathrm{~Hz}$.

$m(\alpha, \theta) \ddot{x}(t)+b(\alpha, \theta) \dot{x}(t)+k(\alpha, \theta) x(t)=u(t)$

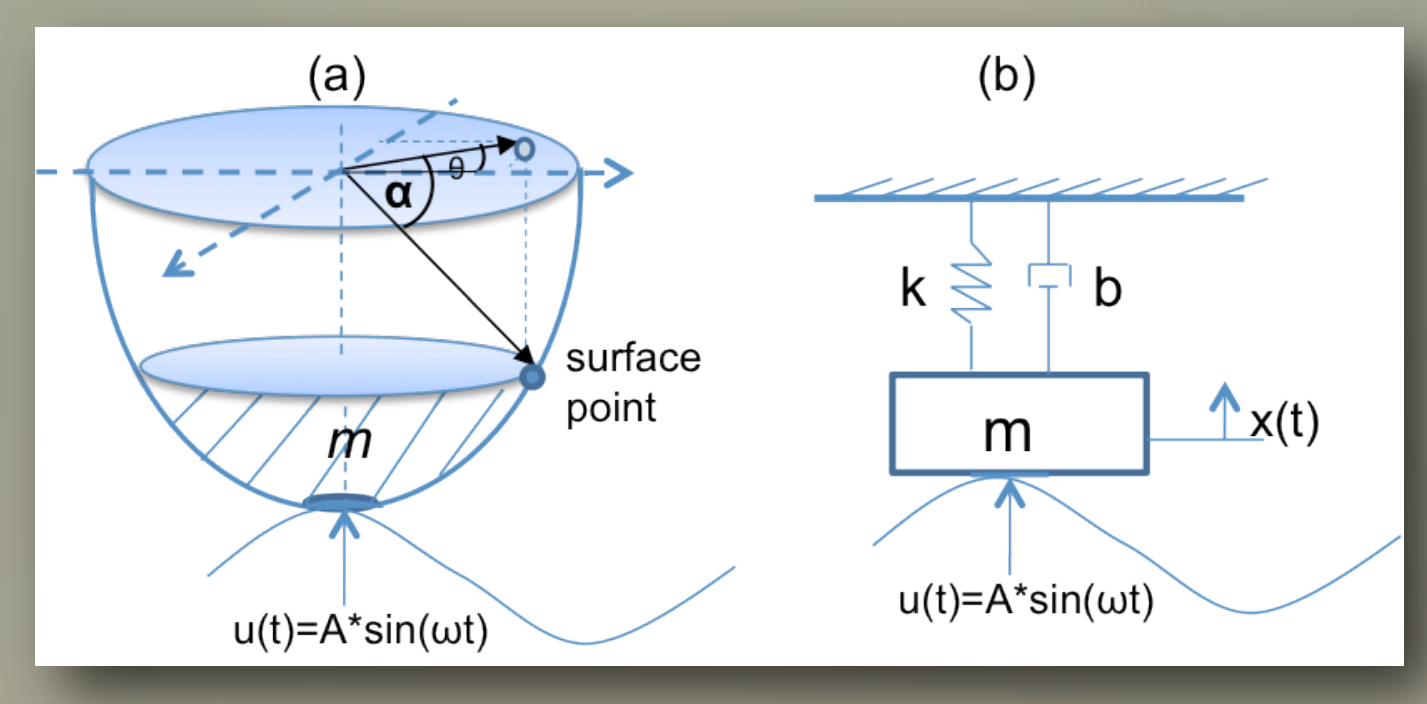

$G_{\alpha, \theta}(j \omega)=\frac{k_{0}(\alpha, \theta)}{\left(\omega_{n}^{2}(\alpha, \theta)-\omega^{2}\right)+2 \xi(\alpha, \theta)}$

$\left(\omega_{n}^{2}(\alpha, \theta)-\omega^{2}\right)+2 \xi(\alpha, \theta) \omega_{n}(\alpha, \theta) j \omega$

$\omega_{n}(\alpha, \theta)=\sqrt{\frac{k(\alpha, \theta)}{m(\alpha, \theta)}}$

$\xi(\alpha, \theta)=\frac{b(\alpha, \theta)}{2 \sqrt{k(\alpha, \theta) m(\alpha, \theta)}}$

2. Mean amplitude $\boldsymbol{A}$ and phase $\boldsymbol{\phi}$ registered for every discrete surface segment $S(\alpha, \theta)$.

3. Transfer function $G_{\alpha, \theta}(j \omega)$ fit to Bode plots of each surface segment; $\omega_{n}(\alpha, \theta)$ and $\xi(\alpha, \theta)$ are identified disregarding data past the first resonant peak.

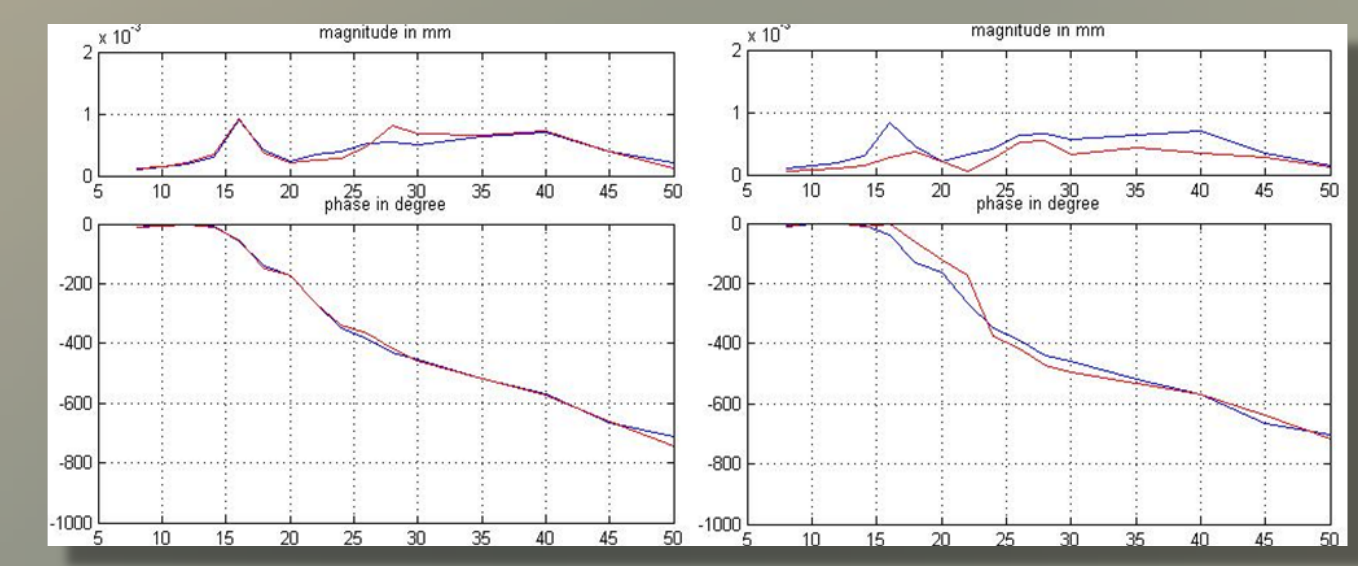

Bode plots of healthy and cancer breast. Shown are the segment opposing the tumor (left), and the area of the tumor inclusion. Blue=healthy, red=cance
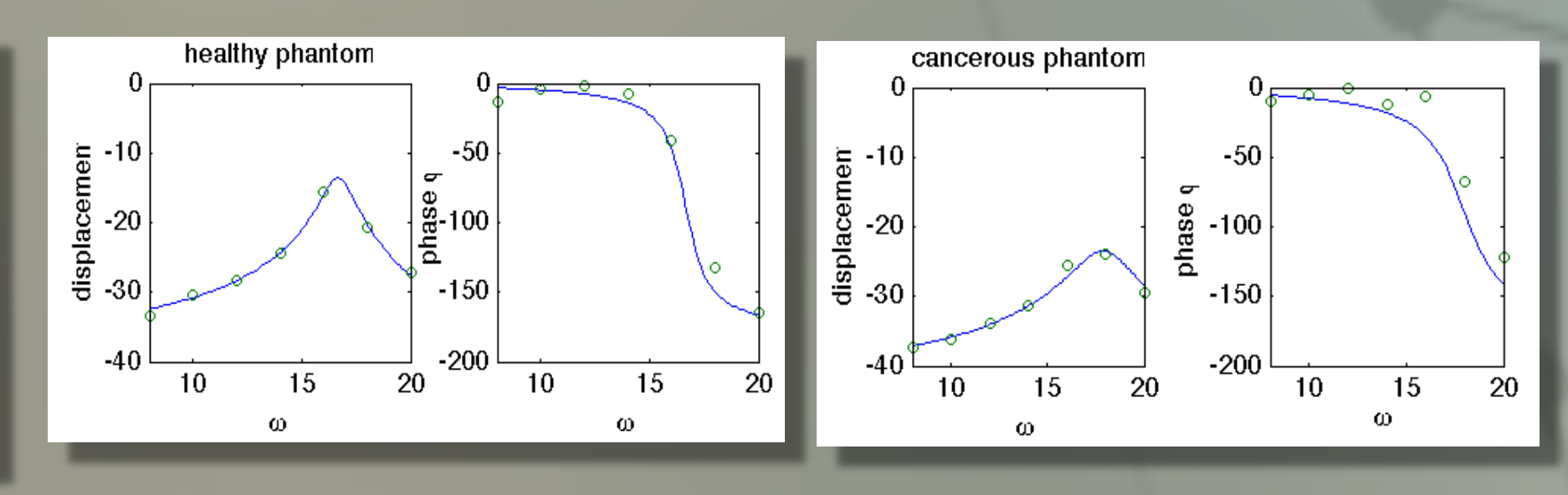

Model fits to the Bode plots at $\alpha=25^{\circ}$ and $\theta=180^{\circ}$ for the healthy (left) and cancerous case (right)

\section{Results}

- Normalized natural frequency and damping ratio plotted for surface segments $S(\alpha, \theta)$

$$
\omega_{n_{\text {nom }}}(\alpha, \theta)=\frac{\hat{\omega}_{n}(\alpha, \theta)}{\min _{\alpha}\left(\hat{\omega}_{n}(\alpha)\right)} \quad \xi_{\text {norm }}(\alpha, \theta)=\frac{\hat{\xi}(\alpha, \theta)}{\min _{\alpha}(\hat{\xi}(\alpha))}
$$

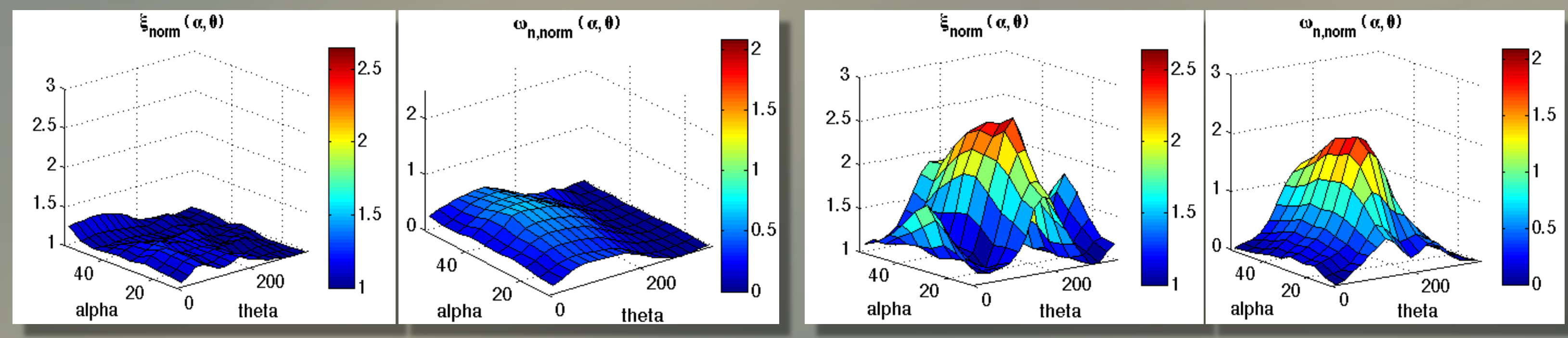

Identified normalized natural frequency and damping ratios for surface segments $S(\alpha, \theta)$ for the healthy phantom (left) and the phantom with a $20 \mathrm{~mm}$ stiffer inclusion (right).

- Clear increase in both parameters seen at location of tumor $\theta=180^{\circ}$

- Size of tumor not identifiable, but presence and angular location

\section{Discussion and Conclusions}

- The proposed minimal modeling approach is able to capture frequency response data from the surface of an oscillating breast phantom.

- Normalized damping $\xi_{\text {norm }}(\alpha, \theta)$ and natural frequency $\omega_{n, \text { norm }}(\alpha, \theta)$ values identified over the surface of the breast show a distinctive difference around the location of the tumor.

- $\theta$ matches the position of the tumor exactly. $\alpha$ differs from the angle of the tumor inclusion, which is due to the asymmetrical geometry of the breast relative to the vibration source.

- Good model fit achieved with single mass model, but improvements expected with more complex modelling approaches.

- In vivo validation required (clinical data collection underway) 